die reducirende Substanz aus dem Eieralbumin in schönen Crystallen rein dargestellt und mit einem schon bekannten kohlehydratähnlichen Körper identificirt worden war, ist es selbstverständlich, dass auch die Derivate dieser beiden Stoffe identisch sein müssen. Glukosamin liefert, wie Tiemann gezeigt hat, Glukosazon. Wenn Blumenthal und Mayer nach unseren Methoden arbeiten wollen, wird es ihnen mit Leichtigkeit gelingen, auch den Stickstoffgehalt und das Rechtsdrehungsvermögen des von ihnen gesuchten Zuckers zu erkennen. Da die Autoren unsere Arbeiten kannten, so ist es uns schwer verständlich, warum sie diese bereits bekannten Thatsachen nicht erwähnten und zu der Vermuthung kamen, es müsste der Beweis für die $\Lambda$ bspaltıng einer Hexose aus dem Eieralbumin erst erbracht werden. Blumenthal und Mayer erwähnen, dass das von Seemann verarbeitete Albumin vielleicht nicht ganz frei von Ovomucoid war, doch erwähnt Seemann ausdrücklich, dass er sein Prüparat sorgfültig von anhaftendem Traubenzucker und Ovomucoid durch wiederholtes Auskochen gereinigt hatte, und er hebt hervor, wie unwahrscheinlich es ist, dass der bedeutende Gelialt des Albumins an reducirender Substanz $(8 \%)$ von einer Beimengung von Ovomucoid lerrührte. Auch Weiss ${ }^{1}$ ) und S. Fränkel, ${ }^{3}$ ) die sich neuerdings mit der Frage nach der reducirenden Substanz aus Eiereiweiss beschäftigt haben, bedienten sich desselben Verfahrens und haben danach ihr Eieralbumin als frei von Ovomucoid aufgefasst. Nur die Thatsache, dass das kohlehydratähnliche Spaltungsprodukt des Eieralbumins identisch war mit dem des Ovomucoids, veranlasste Seemann, die Möglichkeit nicht auszuschliessen, dass auch die Muttersubstanz verwandt oder identiseh war. Diese Möglichkeit wird vielleicht dudurch eliminirt werden können, dass crystallisirtes Albumin als Ausgangsmaterial verwandt wird. Die von Blumenthal und Mayer geübte Reinigungsmethode unterscheidet sich von der unserigen nur dadurch, dass das durch Siedehitze coagulirte Eiweiss nach dem Auswaschen mit Alkalilauge wieder in Lösung gebracht und dann mit Essigsiiure ausgefällt wurde. Man wird den Beweis abwarten inüssen, ob ein so eingreifendes Verfahren zweckmässiger und geeignet ist, ein reineres Eieralbumin darzustellen, und ob aus diesem andere Spaltungsprodukte erhalten werden, als wir unter Anwendung. des bisher üblichen Reinigungsverfahrens gewinnen konnten.

$\mathrm{Ob}$ ausser dem Glukosamin noch andere kohlehydratartige Körper

\section{Ueber die Abspaltung von Zucker aus} Eiweiss.

\section{Bemerkungen zu dem gleichnamigen Aufsatz des Herrn Dr. Paul Mayer} in No. 6 dieser Wochenschrift.

Von Professor Dr. Friedrich Müller und Dr. John Seemann in Marburg.

In dem oben genannten Aufsatz liat Herr P. Mayer gezeigt, dass das Osazon, welches sich aus Eieralbumin nach dem Kochen mit verdünnter Salzsäure gewinnen lässt, die Zusammensetzung und den Schmelzpunkt eines Hexosazons darbietet. Gleichzeitig erschien in den Berichten der deutschen chemischen Gesellschaft und der Pariser Académie des sciences eine Arbeit von Blumenthal und P. Mayer, in welcher die nämliche Thatsache angeführt und dahin erweitert wird, dass es sich um Glukosazon handelt. Die Autoren kommen zu dem Schluss: Damit ist die Abspaltung einer Hexose aus Albumin dargethan." Da durch diese Darstellung. der Glaube erweckt werden könnte, als ob es erst diesen Arbeiten gelungen sei, den oben erwähnten Nachweis zu liefern, so sehen wir uns genöthigt, zur Richtjgstellung Folgendes anzuführen.

Blumenthal und Mayer haben den Nachweis einer Hexosegruppe im Eieralbumin dadurch geführt, dass sie ein Derivat, das Osazon, untersuchten. Dieses indirekten Beweises bedurf es aber heute nicht mehr: Nachdem der eine von uns (Müiller) die aus Mucin abspaltbare reducirende Substanz rein dargestellt und als Glukosamin erkannt hatte, gelang es dem andern (Seemann) mit denselben Methoden diesen Körper auch ats sorgfältig gereinigtem Eieralbumin zu erhalten. Durch Elementaranalyse, durch die specifische Drehung und, was am beweisendsten ist, durch crystallographische Untersuchung und Messung wurde dargethan, dass dieser aus Eieralbumin abspaltbare kolllehydratähnliche Körper identisch war mit dem Glukosamin von Ledderhose. $\left.{ }^{1}\right)$ Die aus Eiereiweis abspaltbare reducirende Substanz ist also gar niclit, wie Pavy, Blumenthal und Mayer vermuthen, ein "Zucker“ im engeren Sinne (Blumenthal und Mayer denken an Galaktose, Glukose, Mannose und Lävulose), sondern die Amidoverbindung einer Hexose. Nachdem

1) F. Müller, Sitzungsbericht der Gesellschaft zur Beförderıng. der gesammten Naturwissenschaften 1896, No. 6 und 1898, No.6. Seemann, Ueber die reducirenden Substanzen, welche sich aus Hühnereiweiss abspalten lassen, Inauguraldissertation, Murburg 1898, abgedruckt im Archiv für Verdauungskrankheiten IV. Unsere Angabe, dass aus Eieralbumin und Mucoid Glukosamin abgespalten wird, hat rasch Bestätigung erfahren, in dem kürzlich erschienenen Jahresbericht für die Fortschritte der Thierchemie findet sich eine Arbeit von Zanetti, der für das Ovomucoid zum selben Resultat kommt, S. Fraenkel konnte unsere Angaben für das von Ovomucoid befreite Eieralbumin bestätigen. im Eiereiweiss vormebildet sind, ist vor der Hand noch nicht zu entscheiden. Nach Blumenthal und Mayer spricht dafür der Umstand, dass neben dem Glukosizon noch Phenylhydrazinverbindungen von niedrigerem Schmelzpunkt, grösserer Löslichkeit und anderem mikroskopischen Aussehen erhalten wurden. Doch ist dieser Befund wenig beweiskräftio, denn solche unreinen, niedrim schnielzenden Osazone werden neben den höher schmelzenden stets und in desto grösserer Menge gefumden, je weniger rein das Ausgangspräparat war. Befreit man das nach der Spaltung von Mucin, Ovomucoid oder Albumin mit $\mathrm{HCl}$ resultirende Filtrat grïndlich von den Albumosen, so behält man ein hohes schmelzendes und besser crystallisirendes Osazon, als wenn man keine derartige Reinigung vorausschickt. Tiemann ${ }^{3}$ ) ist es selbst bei Anwendung reinen Glukosamins nur nach vielen Bemühungen gelungen, reines Glukosazon vom richtigen Schmelzpunkt zu gewinnen, und auch Blumenthal und Mayer geben an, dass sie bisweilen, bei anscheinend reinen Osazonpräparaten, einen Schmelzpunkt von $195^{\circ}$ statt des richtigen von $205^{\circ}$ fanden. $\mathrm{W}^{\top} \mathrm{e} \mathrm{s}^{*}$ ) hat aus Eieralbumin Osazone vom

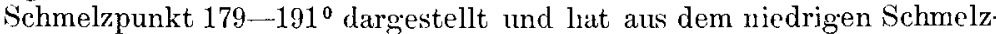
punkt und der (nicht publicirten) Elementaranalyse geschlossen, dass nicht eine Hexose, sondern eine Metlyylpentose vorlag. - Wie wenig Gewicht auf den Schmelzpunkt, die Crystallform, die Löslichlkeit und selbst die Elementaranalyse nicht vollständig reiner Osazone gelegt werden darf, haben wohl alle diejenigen erfahren, die sich eingehender mit diesen Fragen beschäftigt haben.

Pavy ebenso wie Blumenthal und Mayer sind bei ihren Untersuchungen von dem Bestreben ausgegangen, im Eiweissmolekül denjenigen zuckerartigen Atomcomplex abzuspalten, der es erklären soll, dass bei schwerem Diabetes auch in Hunger und bei kohlehydratfreier Kost noch grosse Mengen von Zueker ansgesschieden werden ${ }^{5}$ ).

1) Weiss, Centralblatt für Physiologie XII, 15 .

2) S. Fränkel, Sitzungsbericht der Wiener Akademie der Wissenschaften 1898,107 , II b.

3) Tiemann, Berichte der deutschen chemischen Gesellschaft 17, S. 241 und 19, s. 49.

4) Weiss, l. c. stützt die Annahme, dass atus dem Eieralbumin eine Methylpentose abgespalten werde, ausserdem noch aüf den Ausfall einer Farbenreaction im Destillat. Man wird die ausführliche Publication abwarten müssen, ehe man beurtheilen kann, wie weit den $\Lambda \mathrm{n}$ gaben von Weiss Beweiskraft zuzuerkennen ist. Unsere Untersuchungen stimmen mit seinen Befunden nicht überein

$\left.{ }^{5}\right)$ Es ist allerdings noch nicht sicher erwiesen, dass diese Traubenzuckermengen wirklich nur aus dem Eiweiss der Nahrung oder des Körpers stammen, die Möglichkeit, dass sie aus Fett gebildet werden könnten, ist nocll nicht sicher widerlegt, und vom chemischen Standpunkt würde die Zuckerbildung aus Fett keine grösseren und keine geringeren Schwierigkeiten bieten als die aus Eiweiss; doch sprechen 
Man dachte sich (neucrding:s hält auch L usk ${ }^{1}$ ) an diescr Anschauung fest), dass im „Eiweiss“ des Körpers und der Nahrung ein grosser kohlchydratartiger oder wenigstens stickstofffreier Atomcomplex vorhanden sei, der im Stoffwechsel von den stickstoffhaltigen Gruppen der Eiweissmolekiile abgetrennt und bei Diabetes zu Traubenzucker verwandelt und nicht weiter oxydirt werden sollte. Wir möchten nicht versüumen, nochmals ${ }^{2}$ ) darauf hinzuweisen, dass die Anschaumo nach den ncueren Untersuchungen kaum mehr zulässig ist. Durch den qualitativen und quantitativen Abbau des Eiweissmoleküls, wie er besonders durch Kosscl inaugurirt worden ist, wurde der Nachweis geführt, dass die verschiedenen Proteïnsubstanzen trotz ihrer ziemlich gleichbleibenden elementaren Zusammensetzung eine sehr verschiedene Constitution darbieten, insbesondere lässt sich ein kohlehydratartiger oder sagen wir besser reducirender Bestandtheil durchaus nicht bei allen nativen Eiweisskörpern auffinden, geschweige denn in gleicher Menge nachweisen. Im Caseïn fehlt er zum Beispiel vollkommen, im Muskeleiweiss und im Scrumalbumin ist er anschcinend nur in sehr geringer Menge vorhanden, nur im Eieralbumin erreicht er eine bedeutendere Grösse $\left.(\text { circa } 8 \%)^{3}\right)$. Man ist also eigentlich nicht berechtigt, in Stoffwechselversuchen und ihren Berechnungen das "Eiweiss" als einen stets gleichartigen Stoff einzustellen und in ihm einen kohlchydratartigen Complex als constant vorhanden anzusehen, wie er im Eieralbumin nachgewiesen ist. - Von dem einzigen bisher bekannten kohlehydratähnlichen Spaltungsproduct der Eiweisskörper, nämlich dem Glukosamin, ist es ïberdies noch nicht bekannt, ob es im Organismus in Traubenzucker iibergehen kann. Da die freie Base in wässriger Lösung bei alkalischer Reaction ganz ausserordentlich leicht zersetzlich ist $\left.^{t}\right)$, erscheint es sogar recht wohl möglich, dass sie auch in den Körpersäften rasch zerstört wird.

Aber selbst wenn im "Eiweiss" durchschnittlich ein so hoher Gchalt an reducirender Substanz vorhanden wäre, wie im Eiereiweiss oder selbst im Ovomucoid oder Mucin, so wiüde dies inımer noch nicht hinreichen, um die grossen Zuckermengen zu erklären, von denen nachgewiesen ist, dass sie sich bei schwerem Diabetes aus dem Eiweiss bilden.

Minkowski ${ }^{5}$ ) fand bei Hunden nach Pankreasexstirpation, dass bei Hunger und reiner Fleischkost das Verhältniss des Stickstoffs zum Zucker im Harn fast constant 1:2,8 beträgt, woraus berechnet werden kann, dass anf $100 \mathrm{~g}$ zersetzten Eiweiss ungefähr $45 \mathrm{~g} \mathrm{Zucker} \mathrm{aus-}$ geschieden werden. v. Mering ${ }^{6}$ ) konnte beim Phloridzindiabetes den Nachweis führen, dass auf einen Eiweissumsatz von $100 \mathrm{~g}$ sogar eine Ausscheidung von $60 \mathrm{~g}$ Traubenzucker traf, und diese Angabe ist von $\mathrm{Lusk}^{7}$ ) dahin erweitert worden, dass nach hohen Phloridzingaben bei Hunden im Hungerzustand sowohl als bei ausschlicsslicher Fïtterung mit Fleisch und Leim das Verhältniss des Stickstoffs zur Dextrose im Harn nahezu constant wie $1: 3,75$ bleibt; daraus lässt sich der Schluss ziehen, dass aus $100 \mathrm{~g}$ Eiweiss 58,7 bis $60 \mathrm{~g}$ Zucker gebildet worden scin müssen.

Bei sehwerem menschlichem Diabetes ist bei kohlchydratfreier Kost wiederholt sehon das Verhältniss von N: Traubenzucker im Harn wie $\left.1: 3,2,{ }^{8}\right) 1: 2,9$ ja sogar $1: 3,7^{\circ}$ ) gefunden worden, was mit Minkowski's, v. Mering's und Lusk's Zahlen übereinstimmt.

Ist es nun möglich, dass im Eiwcissmolekül eine diesen Zahlen ent-

eine Reihe gewichtiger Gründe dafïr, dass beim schweren Diabetes des Menschen und beim experimentellen Diabetes der Thiere die Zuckerbildung thatsächlich nur auf Kosten des Eiweiss stattfindet. Bei diesen Diabetesfällen steigt die Zuckerausschcidung mit der Steigerung der Eiweissnahrung, während Fettdarreichung keineZunahme der Zuckerausscheidung erzeugt. Beim Pankreasdiabetes hat Minkowski im Hunger und bei Fleischfütterung ein constantes Verhältniss zwischen der Zuckerausscheidıng und dem Eiweissumsatz gefunden. Bei Phloridzindiabetes konnte v. Mering und nenerdings in einer sehr sorgfältigen Arbeit Lusk nachweisen, dass im Hungerzustand wic auch bei ausschliesslicher Fleisch- und Lcimfiitterung: die Zuckerausscheidung dem Eiweissumsatz genau proportional geht und dass durch Fettzufuhr keine Zunahme, sondern eine Abnahme der Zuckerausscheidung stattfindet.

1) Lusk, Reilly und Nollan, Phloridzindiabetes in dogs. American Journal of Physiology 1898, Vol. I. Ferner: The Chemistry of the proteid molecule. Yale medical Journal, June 1898.

$\left.{ }^{2}\right)$ cf. F. Müller, Allgemeine Pathologie der Ernährung in v. Leyden's Handbuch der Ernährungstherapie Bd. I, S. 188.

3) Aus Ovomucoid lässt sich allerdings eine grössere Menge reducircnder Substanz abspalten, nach Se emann circa $15 \%$, aus dem Mucin des Sputums $34 \%$ und ans dem der Submaxillaris $24 \%$, doch kommen diese Mucine und Mucoide für den Stoffwechsel wohl weniger in Betracht.

4) cf. Breuer. Berichte der deutschen chemischen Gescllschaft 1898 , Bd. XXXI, S. 2193

$\left.{ }^{5}\right)$ Minkowski, Untersuchungen iiber Diabctes mellitus nach Ex stirnation des Pankreas. Leipzig 1893.

$\left.{ }^{6}\right)$ v. Mering, Zeitschrift füir klinische Medicin Bd. XIV, S. 431.

i) Reilly, Nollan und Lusk, American Journal of Physiology 1898 , Vol I.

$\left.{ }^{8}\right)$ Eigene Beobachtuno

9) Rump f, Berliner klinische Wochenschrift 1898, No. 43. sprechend grosse Menge von kohlehydratartigen oder sagen wir auch nur stickstofffreien Stoffen präformirt vorhanden ist?

Herr College Kossel hatte die grosse Liebenswiüdigkeit, mir einige seiner Untersuchungen zur Verfügung zu stellen, in wclchen er die Zersetzungsproducte verschiedener Eiweisskörper nach Spaltung mit Säuren quantitativ bestimmt hatte. Diese Untersuchungen, welche Herr Professor Kossel zum Theil in Gemeinschaft mit Herrn Dr. Kutscher ausführt, sollen später von ihnen ausführlich mitgetheilt werden. Die hier angeführten Zahlen können noch nicht als endgiltige betrachtet werden, sie xenügen aber, um eine Uebersicht über die Vertheilung des Kohlenstoffs auf die versehiedenen Spaltungsproducte der Eiweisskörper zll geben.

$$
15 \% \mathrm{~N} \quad \text { Eieralbumin. }
$$

Spaltung mit $20 \%$ salzsäure 72 Stunden

Gesammtstickstoff $\quad=100$ gesetzt entspricht $355,0 \mathrm{~g}$ Kohlenstoff

darin Hexonbasen

(Arginin, Histidin, Lysin) $=27,0 \mathrm{~N}$

Stickstoff der Mono-

amidosäuren . . . $=50,4 \mathrm{~N} \quad, \quad 237,6 \mathrm{~g} \quad$ (annähernd)

$77,4 \mathrm{~N}$ entspricht $284,2 \mathrm{~g} \mathrm{Kohlenstoff}$

In Form nicht zuckerartiger Verbindungen finden sich also etwa $80 \%$ des gesammten Kohlenstoffs.

$$
18,5 \% \mathrm{~N} \text { Histon. } 52,3 \% \mathrm{C}
$$

Spaltung mit $33 \%$ schwefelsäure 20 stunden.

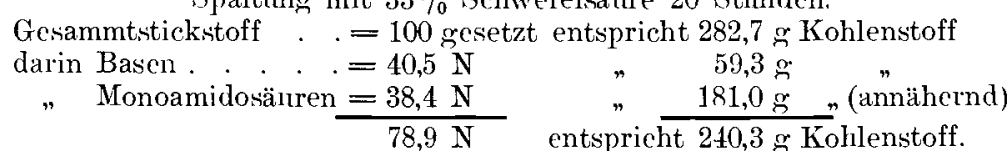

In Form nicht zuckerartiger Verbindungen sind also annähernd $85 \%$

des gesammten Kohlenstoffs vorhanden.

$$
\begin{aligned}
& \begin{array}{l}
15,7 \% \mathrm{~N} \text { Case ï } 53,0 \% \mathrm{C} \\
\text { Gesammtstickstoff } .=100 \text { gesetzt entspricht } 337,6 \mathrm{~g} \text { C. }
\end{array} \\
& \text { darin Basen . . }=23,0 \mathrm{~N}, 41,2 \mathrm{~g} \text { C. }
\end{aligned}
$$

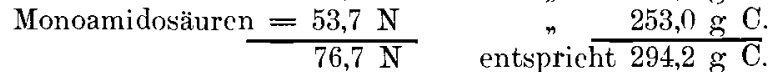

In Form nicht zuckerartiger Verbindungen finden sich also ungefähr $87 \%$ des gesammten Kohlenstoffs.

Unter den Monoamidosäuren ist Leucin, Tyrosin, Glutaminsäure und Asparaginsäıre zu verstehen; von diesen bildet Leucin die weit überwiegende Menge. Unter den stickstoffhaltigen Spaltungsproducten findet sich stets auch Ammoniak (eirca 10\% des Gresammtstickstoffs) sowic cin wechselnder Bruchtheil unbekannter Stoffe, welche ebenfalls noch eine gewisse Menge von Kohlenstoff in Anspruch nehmen; unter eincr dieser letzteren, oben nicht in Rcchnung eingestcllten Gruppen ist auch das Glukosamin zu erwarten.

Diesc Analysen Kossel's zeigen also, dass im Eiweissmolekül durch die nicht zuckerartigen Verbindungen (Leucin, Tyrosin, Glutaminsäure, Asparaginsäure, Arginin, Histidin, Lysin u. a.) bereits 80 bis $87 \%$ des gesammten Kohlenstoffs in Anspruch genommen sind; für dic zuckerartigen Verbindungen bleiben höchstens 20-13\% des Kohlenstoffs übrig, und zwar beim Eieralbumin, das grössere Mengen reducirender Substanzen abspalten lässt, mehr als bcim Cascïn, das davon frei ist. R. Cohnl) glaubte sogar bei der Spaltung des Cascïns durch Salzsäure 91-97,8\% der zur Analyse verwandten Menge in Form von Spaltungsproducten erhalten zu haben, es blieben dann also noch weniger stickstofffreic Körper als möglich ïber.

Nehmen wir den günstigsten Fall, nämlich den des Eicralbumins, wo $20 \%$ des gesammten Kohlenstoffochalts in zuckerartigen Verbindungen vorhanden sein können, so wïrden diese 50 Theilen Zucker entsprechen. Bei einem Kohlenstoffgehalt des Eicralbumins von 53\% wiirde sich daraus berechnen lassen, dass auf $100 \mathrm{~g}$ Albumin höchstens $26 \mathrm{~g}$ Zucker treffen könnten. Für die anderen Eiweissstoffe würde sich aber noch cine geringere Menge von Zucker als möglich ergeben

Diese Zahlen reichen also nicht entfernt hin, um die gewaltigen Zuckermengen zu erklären, wclche sich nach den Berechnungen von Minkowski, v. Mering, Lusk beim experimentellen Diabetes oder auch nach den oben angeführten Beispielen beim Diabetes des Mensehen aus Eiweiss bilden müssen.

Es bleibt also nur übrig, anzunehmen, dass der Zucker, welcher sich bei Diabetes aus Eiweiss bildet, nicht als solcher präformirt im Eiweissmolekiil vorgebildet ist, sondern dass el sich aus stickstoffhaltigen Atomcomplexen dureh theilweise Oxydation und unter $\mathrm{Ab}$ spaltung des Stickstoffs (zum Beispiel der Amidogruppe) bildet..2) Man

I) Rudolph Cohn, Ueber eine quantitative Eiweissspaltung durch Salzsäure. Zeitschrift für physiologisehe Chemie Bd. XXVI, s. 395.

2) Eine ähnliche Anschaumng, dass nämlich der Zucker aus den N-haltigen Eiweissbestandtheilen durch "dic Amidirung“ entsteht, hat auch S. Fränkel in einer soeben erschienenen Arbeit ausgesprochen. Sitzungsberichte der Akademie der Wissenschaften in Wien, mathematisch-naturwissenschaftliche Klasse, Bd. CVII, II, b. 
wird dabei zunächst an diejenigen Spaltungsproducte des Eiweiss denken müssen, die sechs Atome Kohlenstoff aufweisen und welche von Kossel als Hexone bezeichnet worden sind, also die Hexonbasen und vor allem das Leucin. 